\title{
Smart Shoes to Avoid Falls in the Elderly People
}

\author{
Higor Alves Ferreira \\ Pontifical Catholic University of \\ Goiás, \\ PUC Goiás, Goiânia/GO Brazil
}

\author{
Gabrielly Craveiro Ramos \\ Pontifical Catholic University of \\ Goiás, \\ PUC Goiás, Goiânia/GO Brazil
}

\author{
Talles Marcelo Gonçalves de \\ Andrade Barbosa \\ Pontifical Catholic University of \\ Goiás, \\ PUC Goiás, Goiânia/GO Brazil
}

\begin{abstract}
The world's elderly population is overgrowing. It is estimated that, by the year 2050 , the number of older people in society has doubled. One of the biggest problems faced by the elderly population is falling. Therefore, this article proposed a smart wearable shoe to prevent falls in the elderly. Fuzzy logic has been integrated into algorithms to lead with uncertain situations. Thus, it is possible to infer the risks of falling in each case correctly. Black box tests and simulations reveal that wearable technologies can avoid falls.
\end{abstract}

\section{General Terms}

Wearable devices, Fall-prevention technology, Older people.

\section{Keywords}

Fuzzy Logic, Arduino, Smart Shoes.

\section{INTRODUCTION}

With improved quality of life, the number of older people has grown significantly [1]. In 2019, about 1 billion people were over 60 in the world [1]. It is estimated that in 2030 this number will rise to 1.4 billion and 2.1 billion in 2050 [1]. Consequently, the demand for primary care and health services will be more significant [1]. Therefore, it is necessary to seek and invest in contributions that enable healthy aging [1].

One of the public health problems for the world's elderly population is falls [2]. In Brazil, 30\% of the elderly fall at least once a year, and in the USA 16\% [3]. Fall prevention represents a significant challenge for the elderly, family members, communities, and health professionals [2]. Falls in the elderly are diverse, such as individual characteristics and inadequate environment [2] [3]. Most of it happens inside the home, ending up being a hostile and risky environment [4]. Among the most common dangers are low light, objects on the way, pets, among others [4].

In the literature, the area of detection of falls has been widely explored, while prevention has few studies [5]. However, as attractive as the detection of falls is, the best way to reduce falls is to prevent them from happening [5].

Wearable devices are devices embedded in clothing or accessories. Its use has become increasingly popular. They allow users to track and monitor physical activity, nutrition, health records, sleep habits, among others [6]. They can also help users be more active by sending motivational alerts and comparing their statistics with other users and the possibility of reward systems [6]. And due to the potential of portability

and ease of use, wearable devices are receiving more and more attention in the health sector [6].

According to [7], falls can be detected based on wearable sensors with a fuzzy logic system. In a fuzzy model, the outputs are controlled by rules applied to the input variables [7]. Thus, the fuzzy logic allows a greater degree of interpretation of the models, in addition to the possibility of treating heterogeneous types of data [8].

This project aimed to create a wearable device in the shape of a shoe to prevent falls in the elderly. This wearable device uses fuzzy logic as an element for decision making when there is uncertainty about the input data related to the environment. Therefore, it is possible to detect some of the factors that can cause a fall. Consequently, one can estimate the risk of falling for each situation in that environment. If the possibility of a fall occurs, the shoe alerts the user to be more cautious at that moment, thus being able to prevent the fall.

\section{MATERIALS AND METHODS}

\subsection{Requirements Analysis}

The requirements analysis activity was carried out by the Academic League of Physiotherapy team for the Health of the Elderly (LAFISI) [10], which is formed by specialists and students in elderly health. LAFISI group identified some of the possible factors responsible for the falls of the elderly during their walks. Thus it is possible to determine a situation of the potential risk of falling and trigger an alert as a form of intervention. Consequently, the sign can increase the user's attention, preventing falls.

\subsection{Solution Analysis}

Given the requirements, it was proposed the construction of a shoe using embedded systems. Where an Arduino Nano microcontroller (ATmega 328), two proximity sensors (HCSR04), 1 module with a water sensor, 1 module with a light sensor, one force sensor (Fsr 402), one buzzer, and one vibration motor.

The proximity sensor frontal was used to detect the obstacles in front of the user. The proximity sensor lateral was used to detect the barriers in lateral the user. The light sensor to inform data about the light level in the environment. The water sensor delivers data on the presence of water in the ground, allowing you to estimate whether the floor can be slippery. The force sensor was used to measure the force applied by the elderly in the shoe. The vibration motor is the primary alert, and the buzzer the secondary alarm. And, to be able to work with heterogeneous variables, Fuzzy Logic was used.

Another critical aspect of being considered was whether the system would be used indoors or outdoors. Most falls happen to be used indoors. It implies considering shorter distances to activate the alert.

\section{PROTOTYPE}

For the prototype's construction, it was necessary to implement both the system's hardware and software. 


\subsection{Software Implementation}

Arduino IDE and the JAVA programming language were used for software development. For the implementation of Fuzzy Logic, the eFLL library [9] was used. The Arduino IDE interface itself can include it in the library manager.

The fuzzy logic system was used for two reasons. The first is the possibility of working with heterogeneous variables, that is, variables that are quantified in different ways. The second is due to the uncertainty about the output for each input value received by the sensors. Therefore, the fuzzy system ended up being relevant to the implementation of this project.

Due to the social isolation generated by covid-19, the software calibration was performed for the project author's home's internal environment.

The frontal proximity sensor is responsible for measuring the distance from objects in front of the user. Three states were determined for the frontal space near, medium, and far. Figure 1 shows the membership functions for the three states where the length in centimeters $(\mathrm{cm})$ is on the $\mathrm{x}$-axis, and the y-axis the membership for this distance. The maximum value considered for the frontal space was $120 \mathrm{~cm}$.

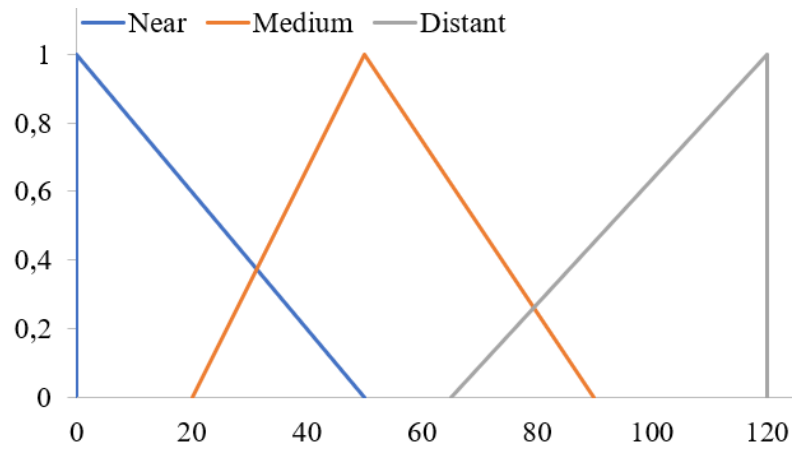

Fig 1: Front proximity sensor ( input variable)

The lateral proximity sensor is responsible for measuring the distance from objects in the lateral of the user. Three states were determined for the lateral distance near, medium, and far. Figure 2 shows the membership functions for the three states where the length in centimeters $(\mathrm{cm})$ is on the $\mathrm{x}$-axis, and the $y$-axis the membership for this distance. The maximum value considered for the lateral space was $60 \mathrm{~cm}$.

Figure 3 shows the pertinence curves used for the luminosity sensor. The $\mathrm{x}$-axis shows the intensity of light in the environment measured in lux, and the y-axis shows the pertinence for each value. Three states were defined to quantify the luminosity of the dark, light, and very bright environment.

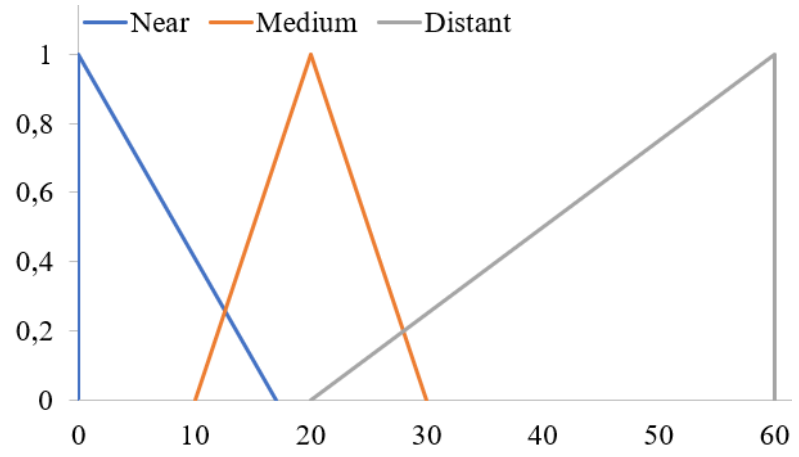

Fig 2: Lateral proximity sensor ( input variable)

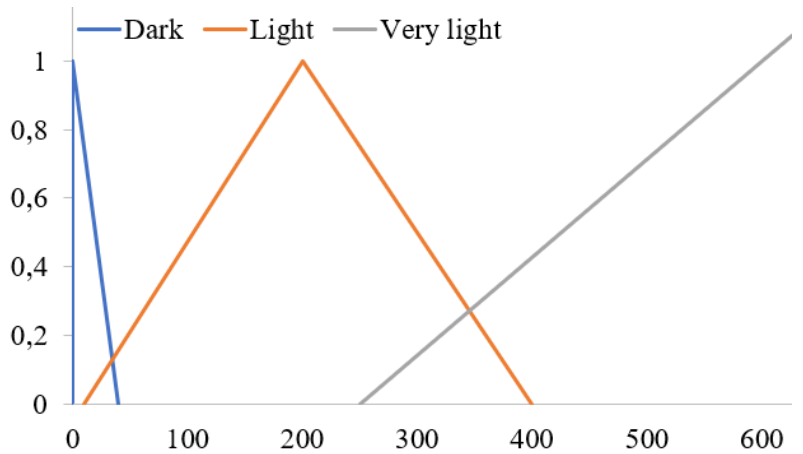

Fig 3: Light sensor ( input variable)

The pertinence curves defined for the water sensor are shown in Figure 4. Only two states have been represented: the absence of water and the presence of water, therefore, a binary cloudy composition. Only two states are because, even on a floor with little water, the risk of slipping and falling is high.

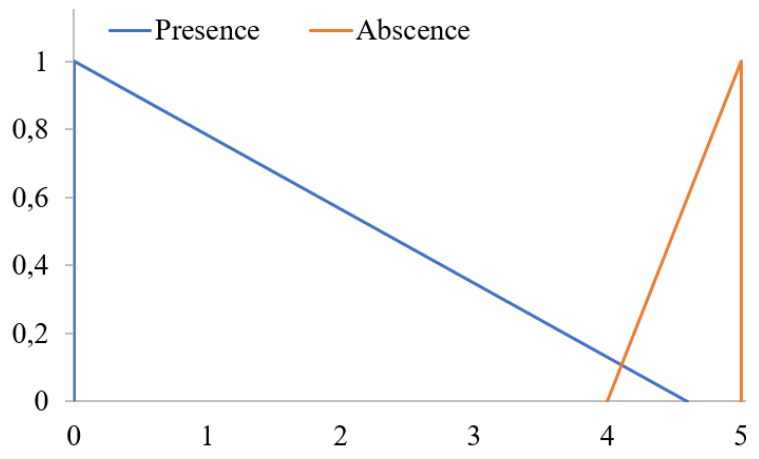

Fig 4: Water sensor (input variable)

The fuzzy rules are shown in Table 1. They are responsible for defining the system output for each data set used in the system input. These rules were determined with the help of a fall specialist. In the first field of the table is the identification of fuzzy control. In the second field of the table is the condition for the rule to be executed. The eFLL library allows a maximum of two conditions per rule. Therefore, rules for the fuzzy system were defined with only one or two states. And in the third field of the table is the output that must be given by the system.

The output variable of the fuzzy system is shown in Figure 5. It defines the possible outputs of the fuzzy system. The fuzzy system output is used to determine the risk of falling at that time.

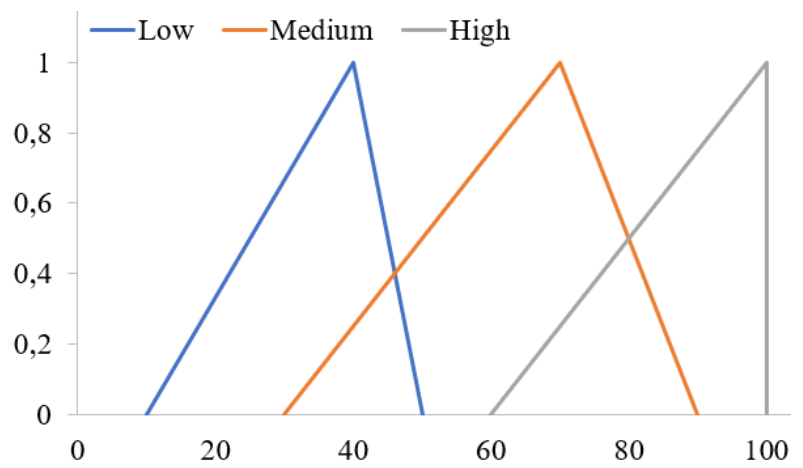

Fig 5: Alert intensity (output variable) 
Table 1. Fuzzy rules

\begin{tabular}{|c|c|c|}
\hline $\mathbf{N}^{\mathbf{o}}$ & Rule & Output \\
\hline 1 & Front proximity: distant & low \\
\hline 2 & Front proximity: near & high \\
\hline 3 & $\begin{array}{l}\text { Front proximity: medium } \\
\text { OR } \\
\text { Lateral proximity: near }\end{array}$ & medium \\
\hline 4 & Lateral proximity: medium & low \\
\hline 5 & $\begin{array}{c}\text { Light: very light } \\
\text { AND } \\
\text { Front proximity: medium }\end{array}$ & low \\
\hline 6 & $\begin{array}{l}\text { Light: very light } \\
\text { AND } \\
\text { Front proximity: near }\end{array}$ & medium \\
\hline 7 & $\begin{array}{c}\text { Light: light } \\
\text { AND } \\
\text { Water: presence }\end{array}$ & high \\
\hline 8 & $\begin{array}{l}\text { Light: very light } \\
\qquad \text { AND } \\
\text { Water: presence }\end{array}$ & medium \\
\hline 9 & Light: dark & high \\
\hline
\end{tabular}

The operation of the code is shown in Figure 6. Initially, the environment is configured. Then, there is an interruption that is activated by time. As shown in Figure 7, this interruption is responsible for reading the environment data with the sensors every $200 \mathrm{~ms}$. Then, the algorithm starts the loop. Afterward, the values that were read by the sensors are assigned to the input variables of the fuzzy system. Soon after, the information is fuzzified according to the rules presented in Table 1. In this step, the inference is made between the fuzzy system's input variables' pertinence functions and the set of rules to generate the output. In the defuzzification stage, a quantifiable result is produced, that is, mapped to clear values. The returned value is a numerical value that is quantified between 0 and 100. This value represents the risk of a fall that the environment may offer at that moment. Therefore, in the next step, the fuzzy system's output value is used as an input parameter for the vibration motor. Thus, the engine can be started with a speed corresponding to the risk of falling, consequently producing the alert at the expected intensity. After the machine is activated with a power greater than or equal to the average, a check is made. During the examination, the force's value exerted on the shoe before and after the alert is compared. Usually, when the person is more attentive, the pressure exerted is more significant, so this comparison is made to reinforce the danger of falling if necessary. Then, when triggering the alert, if the new force exerted on the shoe is less than the first, the bell will ring three times quickly to reinforce the danger of falling.

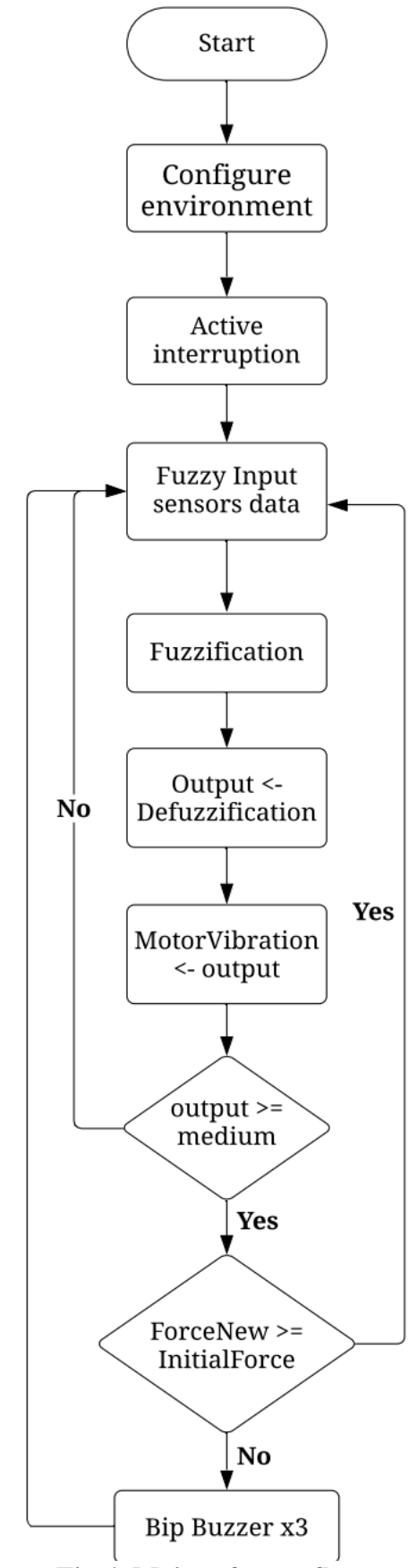

Fig 6: Main software flow

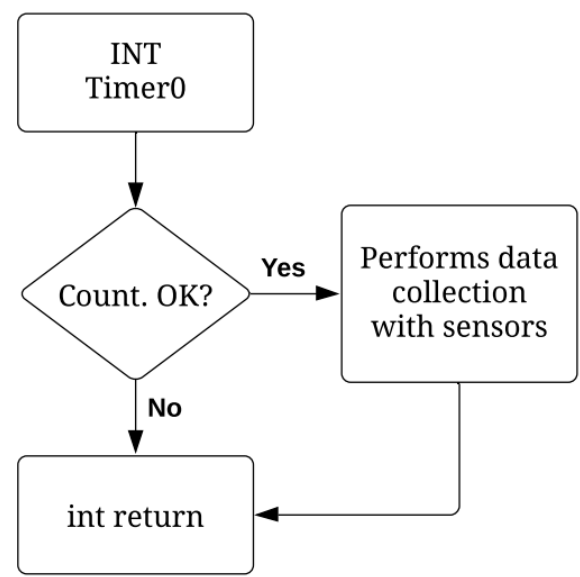

Fig 7: Timer interrupt flow 


\subsection{Hardware Implementation}

The hardware was assembled, as shown in Figure 8. After, it was integrated into a shoe shown in Figure 9. A typical battery (MN-1604) was used to power the hardware connected to a linear voltage regulator (LM7805).

Two galvanized wires have been integrated into the water sensor's sole shoe to work in Figure 10b. The pressure sensor was installed below the heel position, Figure 10a.

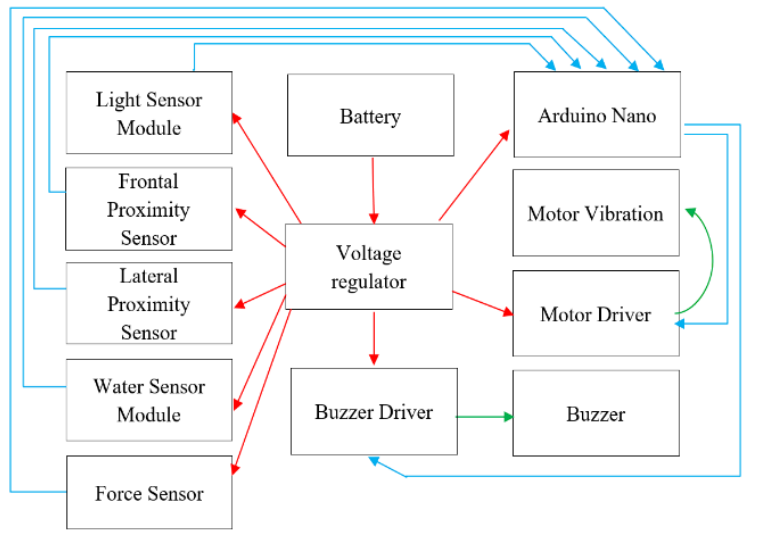

Fig 8: Hardware Block Diagram

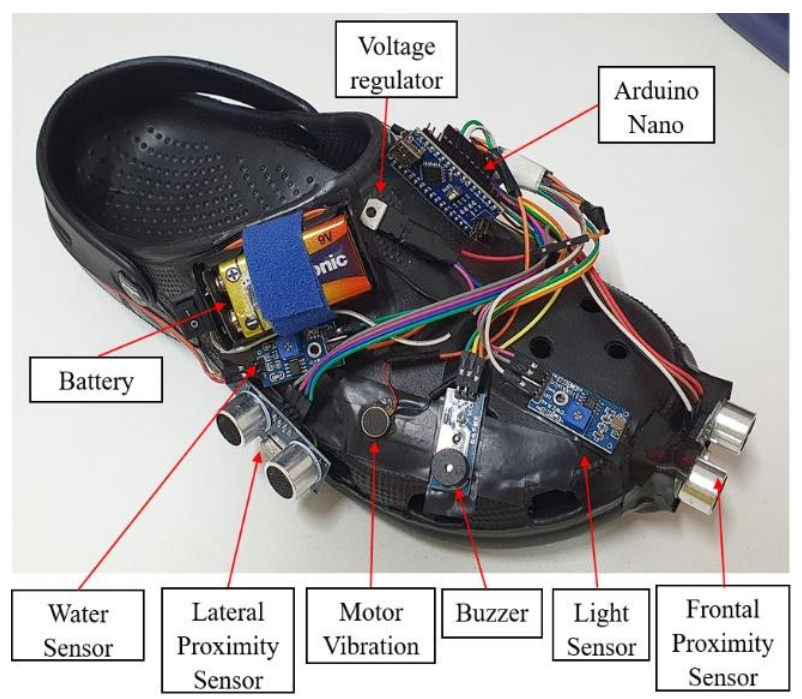

Fig 9: Smart Shoe Prototype

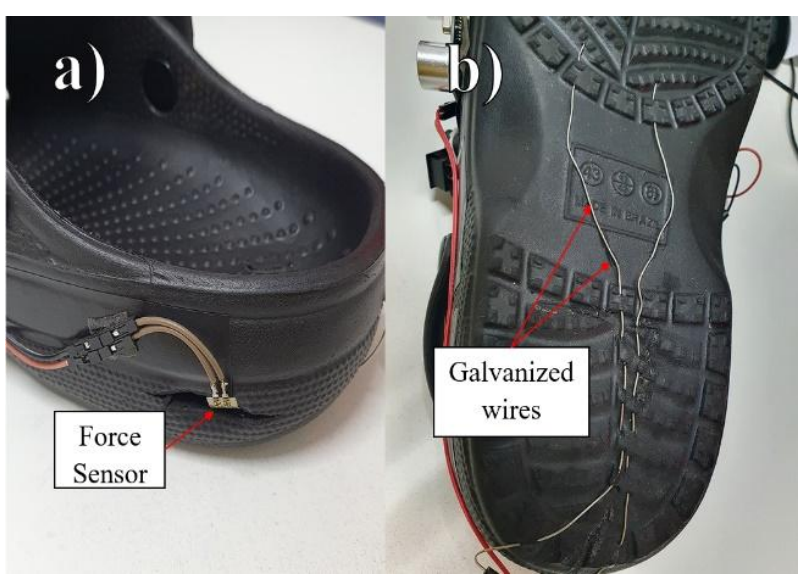

Fig 10: Water and Force Detectors

\subsection{White and black-Box Tests}

White box tests were performed to check for possible logic errors. The microcontroller was connected to a serial port to serve the white box tests. Thus, it was possible to monitor the updated values in the variables at each step of the code, as shown in figure 11. As a result, concise and error-free software was obtained.

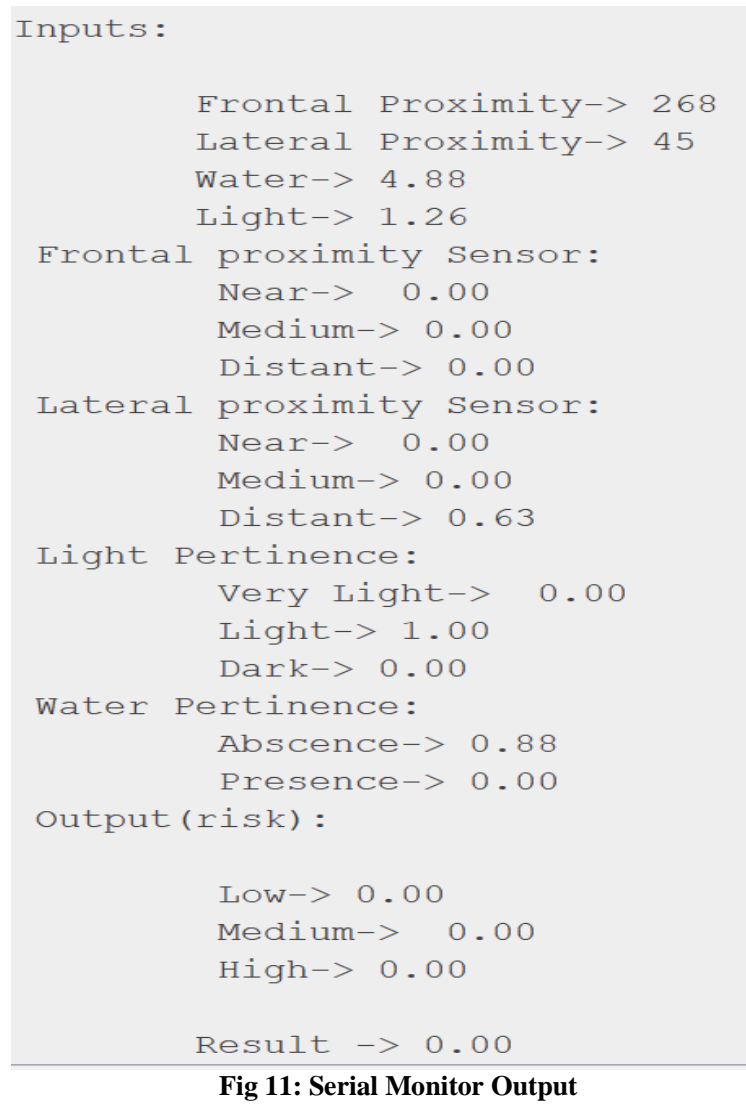

After completing the coding, black-box tests were carried out to ensure that the system shows results consistent with the proposed solution. Each sensor and actuator was tested individually to ensure that they worked as expected to perform the black-box tests. Then, the responses delivered by each of the conditions of the fuzzy system were tested and monitored. During the tests, the correct answer was verified for each of the implemented fuzzy set rules.

\section{RESULTS AND DISCUSSIONS}

This article proposed the development of an intelligent wearable system capable of preventing falls in the elderly. A prototype was built using shoes, sensors, and a microcontroller to meet the requirements (presented in Section 2).

Due to the significant growth of the elderly population, many research lines seek to increase the quality of life and health of the elderly. However, in the area of falls, most works are related to the detection of falls after their occurrence because the faster the service, the less damage will be caused. However, the best way to reduce damage from falls to the elderly population is to prevent them from happening.

For the composition of the results, some tests were carried out with the prototype. As a test protocol for the fuzzy system, each of the rules shown in Table 1 was simulated. Then, the shoes were worn for about an hour to observe each situation that offered the risk of falling. 


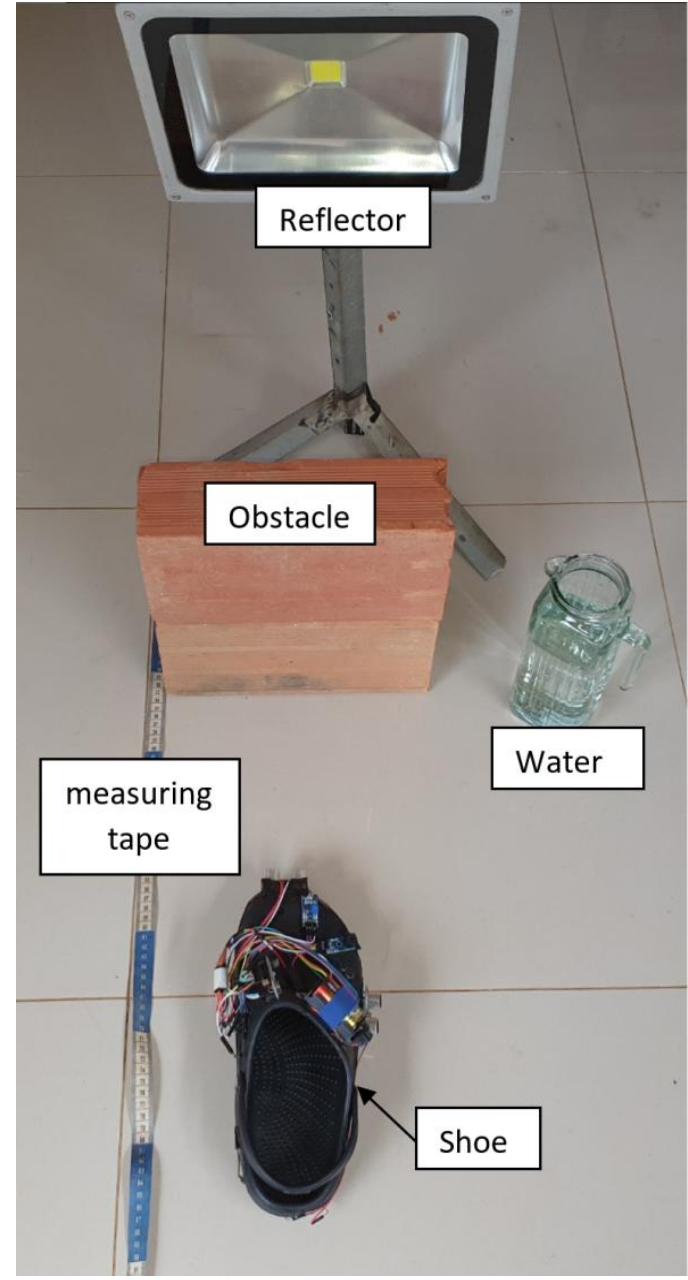

Fig 12: Test objects

Some of the objects used to perform the tests are shown in Figure 12. Where an obstacle, a tape measure, a light reflector, and water.

Initially, an obstacle and a measuring tape were placed in an environment. The footwear has approached the block to measure the distance of the frontal proximity sensor. It was found that when space was between the values determined for the distance away, the shoe vibrated with low intensity, according to rule 1 . As the obstacle approached, the vibration's power increased, passing correctly between the levels of intensity, medium, and high. In the approach, only the lateral distance sensor, the intensity of the vibration was average when it was close and low when it was far. Thus, satisfying rules 1 to 4 .

As the rules from 5 to 9 depending on the light's intensity, it was necessary to add a lux meter and a reflector with adjustable brightness to the previous circuit. When the frontal distance was near and, the light intensity was high, the shoe vibrated with medium intensity by maintaining the light intensity and moving the shoe away until reaching a medium distance. When stepping on a wet floor with the light intensity, the shoe vibrated with high intensity. When stepping on a wet floor with very bright light intensity, the shoe vibrated with medium intensity. And finally, in dark environments or with low light intensity, the shoe shook with high intensity. Thus, satisfying the rules of 5 to 9 .

The next activity evaluated the feedback subsystem when triggering the vibration alert at a medium or higher intensity.
When the force exerted on the shoe was more significant than the first step after activating the alert, the shoe continued to vibrate without an audible alarm. However, when the shoe force was less than that of the first step, the shoe bell rang three times quickly.

In the end, several objects were strategically placed in an indoor environment to offer the risk of falling. In this circuit, the shoe was used for approximately one hour. During use, the shoe was able to issue the alerts correctly. It was able to detect obstacles, animals, wet floors, and low lighting. With that, it was possible to conclude that the shoe is easy and intuitive to use. And it can also promote the increase of the user's attention so that he can be more cautious in risky situations, thus preventing falls.

\section{CONCLUSIONS}

This article proposed the construction of an intelligent system. Which aims to prevent and reduce falls in the elderly. As previously seen, it was found that most falls occur within the elderly's own home. Some of the factors responsible for these falls are obstacles on the way, slippery floors, insufficient lighting, and pets. What makes the solution built as a tool that can be extremely relevant for the elderly. The shoe can easily detect some of these factors. And if there is a risk of falling, the shoe can trigger an alert. Thus, alerting the elderly that there is a risk of falling at that moment. So the shoe ends up helping to prevent a possible fall.

One of the essential aspects that makes this work relevant is applying this type of footwear, which detects objects, to prevent falls in the elderly. Another relevant part is the ease of use of the shoe. One of the contributions of this work, about the majority, is that it aims at avoiding fall before it occurs.

It is crucial to test smart shoes in the laboratory and with older people in future work. Thus, one can verify the shoe's effectiveness in preventing falls and accepting this population to the smart shoe. Such tests were not possible due to the social isolation that had a reason to avoid the contagion and spread of covid-19. Also, calibrations and adjustments must be made to the shoe to work well in different environments. Another exciting development for this work would be implementing a system for detecting falls through web servers. Thus, it would be possible to integrate smart shoes with other intelligent devices. Consequently, the system would be able to detect and prevent falls at the same time.

\section{ACKNOWLEDGMENTS}

The authors would like to thank colleagues Lucas Macedo and Vitor de Almeida and also members of the LAFISI group for their help and support in the development of the work

\section{REFERENCES}

[1] World Health Organization. (2020, December 30). Aging. Available from: https://www.who.int/healthtopics/ageing\#tab=tab_1.

[2] Pimentel, W. R. T., Pagotto, V., Stopa, S. R., Hoffmann, M. C. C. L., Andrade, F. B. de, Souza Junior, P. R. de, Lima-Costa, M. F., \& Menezes, R. L. de. (2019). Falls among Brazilian older adults living in urban areas: ELSIBrazil. Revista De Saúde Pública, 52(Suppl 2), 12s. https://doi.org/10.11606/s1518-8787.2018052000635.

[3] Gonçalves, Andréa Kruger, Teixeira, Adriane Ribeiro, Valentini, Nádia Cristina, Vargas, Amanda Suely Rodriguez, Possamai, Vanessa Dias, \& Martins, Valéria Feijó. (2019). MULTICOMPONENT PHYSICAL ACTIVITY PROGRAM: STUDY WITH FALLER 
AND NON-FALLER OLDER ADULTS. Journal of Physical Education, 30, e3077. Epub December 20, 2019.https://dx.doi.org/10.4025/jphyseduc.v30i1.3077.

[4] Nooruddin, S., Milon Islam, Md., \& Sharna, F. A. (2020). An IoT based device-type invariant fall detection system. Internet of Things, 9, 100130. https://doi.org/10.1016/j.iot.2019.100130.

[5] Akm Jahangir Alam Majumder, Ishmat Zerin, Sheikh Iqbal Ahamed, and Roger O. Smith. 2014. A multisensor approach for fall risk prediction and prevention in the elderly. SIGAPP Appl. Comput. Rev. 14, 1 (March 2014),

$41-52$. DOI:https://doi.org/10.1145/2600617.2600621

[6] Farivar, S., Abouzahra, M., \& Ghasemaghaei, M. (2020). Wearable device adoption among older adults: A mixedmethods study. International Journal of Information Management, 55 , 102209. https://doi.org/10.1016/j.ijinfomgt.2020.102209.

[7] Bhavesh Pandya, Amir Pourabdollah, and Ahmad Lotfi.
2020. Fuzzy logic web services for real-time fall detection using wearable accelerometer and gyroscope sensors. In Proceedings of the 13th ACM International Conference on Pervasive Technologies Related to Assistive Environments (PETRA 20). Association for Computing Machinery, New York, NY, USA, Article 54, 1-7. DOI: https://doi.org/10.1145/3389189.3397989.

[8] S. Spolaor, M. S. Nobile, G. Mauri, P. Cazzaniga and D. Besozzi, "Coupling Mechanistic Approaches and Fuzzy Logic to Model and Simulate Complex Systems," in IEEE Transactions on Fuzzy Systems, vol. 28, no. 8, pp. 1748-1759, Aug. 2020, DOI: 10.1109/TFUZZ.2019.2921517.

[9] Arduino_eFLL_Library. Available in: < https://github.com/zerokol/eFLL>.

[10] LAFISI - Liga Acadêmica de Fisioterapia na Saúde do Idoso. Available in: < https://sites.google.com/site/fisioterapiasaudedoidoso/>. 